\title{
Prevention of Bone Loss in Type I Diabetic Osteoporosis Mice by Hypoxia Mimicking Agent Deferoxamine
}

\author{
Zhang Dong ${ }^{1}$, Zhao Peng ${ }^{2}$, Jin Jia ${ }^{3}$, Jiang Xifeng ${ }^{3}$, Song Honghui ${ }^{3}$, Jia Peng ${ }^{3 *}$, Xu Youjia ${ }^{3}$ and Deng Lianfu ${ }^{4}$ \\ ${ }^{1}$ Senior Ward, the Second Affiliated Hospital of Soochow University, Jiang Su Suzhou, China \\ ${ }^{2}$ Intensive Care Unit, the Second Affiliated Hospital of Soochow University, Jiang Su Suzhou, China \\ ${ }^{3}$ Department of Orthopeadics, the Second Affiliated Hospital of Soochow University, Insitute for Osteoporosis Research of Soochow Universtiy \\ Suzhou, China \\ ${ }^{4}$ Shanghai Ruijin Hospital, Shanghai Institute of Traumatology and Orthopaedics, Shanghai Key Laboratory for Prevention and Treatment of \\ Bone and Joint Diseases with Integrated Chinese-Western Medicine, Shanghai, China \\ *Corresponding Author: Jia Peng, Department of Orthopeadics, the Second Affiliated Hospital of Soochow University, Insitute for Osteo- \\ porosis Research of Soochow Universtiy Suzhou, China.
}

Received: November 05, 2019; Published: November 27, 2019

DOI: 10.31080/ASOR.2019.02.0126

\begin{abstract}
Objective: To observe bone mineral density, bone volume, bone architecture, bone strength and bone metabolism in type I diabetic osteoporosis mice treated by hypoxia mimicking agent—Deferoxamine/DFO.

Method: Type I diabetic mice models were established by injections of streptozotocin. The mice were randomly divided into three groups: control group (normal mice), diabete group (diabetic mice+saline), diabete + DFO group (diabetic mice + DFO). Micro-CT was used to analyse the bone mineral density, bone volume, bone architecture and bone strength of the trabecula in the distal part of femurs. Three point bending test was carried out to evaluate the bone strength. Haematoxylin and eosin (HE) staining was performed to view the alteration in the number of osteoblasts. Real-time PCR was used to detect the mRNA alteration of Runx-2, Osteoclacin, TRAP in tibias. Western blot was used to detect the protein expression alteration of in HIF-1 $\alpha$ and VEGF in tibias.

Results: There was a decrease in bone mineral density, bone volume, bone strength as well as deteriorated trabecular microarchitecture in diabetic mice compared to control group, which were partly restored by DFO treatment. Moreover, DFO treatment increased the number of osteoblasts, mRNA expression levels of Runx-2, Osteoclacin, TRAP, and protein expression levels of HIF-1 $\alpha$ and VEGF.

Conclusion: Bone loss was partly prevented by DFO treatment in type I diabetic osteoporosis mice, which might be ascribed to increased bone formation via stimulative hypoxia inducible factor singnaling pathway.

Keywords: Hypoxia Mimicking Agent; Deferoxamine; Osteoporosis; Type I Diabete
\end{abstract}

\section{Introduction}

Patients with type 1 diabetes often have abnormal bone metabolism, often presenting as osteoporosis, prone to fragility fractures, and post-fracture healing disorders [1,2]. The pathogenesis of osteoporosis in type 1 diabetes is complicated, and it is related to various factors such as absolute insulin deficiency, decreased osteocalcin content, and hyperglycemia [2-5]. Previous studies by the experimental team have shown that osteoporosis in type 1 diabetic mice is associated with down-regulation of hypoxia-inducible signaling pathways in bone [2]. The hypoxia-inducible factor information pathway is a key pathway for conjugated bone formation and angiogenesis. The results of this research team show that activation of hypoxia signaling pathway can promote bone development and partially prevent osteoporosis in aged mice and ovariectomized mice. To promote osteoporotic bone defect repair and fracture healing [6-8]. Hypoxic mimetic compounds are a class of compounds that activate hypoxic signaling pathways under normoxic conditions, including Deferoxamine (DFO), cobalt chloride, dimethylhexanoyl glycine, etc. The most commonly used is DFO. The role of DFO in inducing angiogenesis and accelerating bone remodeling has been confirmed in several experiments [9-12].

The experiment attempted to use DFO to intervene in a mouse model of type 1 diabetes to observe the changes of bone mineral density, bone mass and bone metabolism in mice after activation of hypoxia signaling pathway, and to find a new method for prevention and treatment of osteoporosis in type 1 diabetes. 


\section{Materials and Methods}

Experimental animals

Thirty-four male C57BL/6J mice (body weight $25 \mathrm{~g}$ ) of 10 weeks old were purchased from Shanghai Slack Laboratory Animal Co., Ltd.Raised in the animal room of the Shanghai Institute of Trauma and Orthopaedics (animal license number SYS201521), maintaining a temperature of 23 degrees Celsius, all mice were free to feed water.

\section{Grouping}

After 2 weeks of environmental adaptation, mice were randomized. The experiment was divided into control group, diabetes group, diabetes + DFO group, with 10 mice in each group. Diabetic mouse model was prepared by intraperitoneal injection of $50 \mathrm{mg} /$ kg streptozotocin (Sigma, St. Louis, MO, dissolved in physiological saline). The control group was only injected with normal saline; streptozotocin was injected continuously for 5 times, after the injection was completed. The mice were fasted to water on the evening of 3 days, and the tail blood was taken from the tail on the 4th day to detect blood sugar. The blood glucose of more than $300 \mathrm{mg} /$ $\mathrm{dL}$ was successfully established as a mouse model of type 1 diabetes. Subsequently, the diabetic + DFO group received $20 \mathrm{mg} / \mathrm{kg} /$ day DFO (Sigma, St. Louis, MO, dissolved in physiological saline) intraperitoneal injection for 2 months, and the control group and the diabetic group received only intraperitoneal injection. brine.

\section{Specimen collection}

After 2 months, mice were sacrificed by intraperitoneal injection of an excess volume fraction of $2 \%$ sodium pentobarbital. The left femur of each group was used for Micro-CT analysis; the right femur was wrapped with wet gauze containing saline, stored in a refrigerator at $4{ }^{\circ} \mathrm{C}$, and used for three-point bending on the second day; It was fixed in $4 \%$ paraformaldehyde for hematoxylin-eosin staining. After the mouse was immersed in the right side of the tibia, it was divided into 2 portions and stored in Trizol and RIPA for quantitative PCR and Western blot detection.

\section{Micro-CT detection and analysis}

A full femur scan with a resolution of $8 \mathrm{um}$ using the GE eXplore Locus SP Micro-CT. The scanned image was reconstructed and analyzed in three dimensions using Microview2.2 image analysis software. The three-dimensional reconstructed image of the region of interest was derived from the 100-layer image below the distal femoral growth plate. Computer automated analysis obtained indicators: bone mineral density (BMD), bone mass/tissue volume (BV/TV), trabecular bone number (Tb.N), trabecular thickness (Tb.Th), trabecular bone separation (Tb) .Sp).

1.5 three-point bending testThe femur was taken out from the refrigerator at $4{ }^{\circ} \mathrm{C}$ and allowed to stand at room temperature for $1 \mathrm{~h}$. A three-point femoral bending test was performed using an
Instron 5569 material testing system. The femoral condyle was placed face up on a two-point stand with a distance of $6 \mathrm{~mm}$ between the two points, followed by loading and a load speed of 10 $\mathrm{mm} / \mathrm{min}$. The indicators obtained include: maximum load, maximum pressure, and fracture energy.

\section{Hematoxylin-eosin staining}

The specimens were fixed in $4 \%$ paraformaldehyde for 48 $\mathrm{h}$, then immersed in 10\% EDTA solution for decalcification for 1 month, and the decalcification solution was changed twice a week. After the specimen was rinsed overnight, the automatic dehydrator was dehydrated and embedded in paraffin. After embedding, the wax blocks were frozen overnight in a refrigerator at $-20^{\circ} \mathrm{C}$. Serial sections of $5 \mu \mathrm{m}$ thickness were then made using a Leica tissue microtome. Tissue sections were placed on a release slide containing polylysine and sectioned in a $60^{\circ} \mathrm{C}$ incubator overnight. The sections were then subjected to hematoxylin-eosin staining, xylenesealed, and photographed under a microscope.

\section{Quantitative PCR detection}

The total RNA of the tibial bone tissue was extracted, and $1 \mathrm{ug}$ of RNA was used for reverse transcription of cDNA. The cells were incubated on a PCR incubator at $25^{\circ} \mathrm{C}$ for 10 minutes, $42^{\circ} \mathrm{C}$ for 60 minutes, and $70^{\circ} \mathrm{C}$ for 10 minutes, and the reaction was stopped on ice. Subsequently, 1 ul of cDNA was added, and primers, SYBR ${ }^{\circledR}$ Green, ROX II, cDNA template, and ddH2O were added for PCR amplification. The RT-PCR reaction conditions were as follows: $94^{\circ} \mathrm{C}$ for 5 minutes, $94^{\circ} \mathrm{C}$ for $30 \mathrm{~s}, 55^{\circ} \mathrm{C}$ for $30 \mathrm{~s}$, and $72^{\circ} \mathrm{C}$ for $30 \mathrm{~s}$. After 35 cycles of this cycle, the reaction was terminated at $72^{\circ} \mathrm{C}$ for 5 minutes. PCR Reverse Transcription Kit (Takara, Dalian, China).

\begin{tabular}{|l|c|c|}
\hline \multicolumn{1}{|c|}{ Gene } & $\begin{array}{c}\text { Upstream Primer } \\
\text { (Bp) }\end{array}$ & $\begin{array}{c}\text { Downstream Primer } \\
\text { (Bp) }\end{array}$ \\
\hline Runx2 & $\begin{array}{c}\text { 5'-GTGTCACTGCGCT- } \\
\text { GAAGAGG-3'[26] }\end{array}$ & $\begin{array}{c}\text { 5'-GACCAACCGAGTCATT- } \\
\text { TAAGGC-3'[22] }\end{array}$ \\
\hline Osteocalcin & $\begin{array}{c}\text { 5'-TGCTCACTCTGCT- } \\
\text { GACCCTG-3'[20] }\end{array}$ & $\begin{array}{c}\text { 5'-TTATTGCCCTCCT- } \\
\text { GCTTG-3'[18] }\end{array}$ \\
\hline \multirow{2}{*}{ TRAP } & $\begin{array}{c}5^{\prime} \text {-GCCCTTACTACC- } \\
\text { GTTTGC-3' }[20]\end{array}$ & $\begin{array}{c}\text { 5-TCTCGTCCTGAA- } \\
\text { GATACTGC-3 [20] }\end{array}$ \\
\hline \multirow{2}{*}{-actin5 } & $\begin{array}{c}\text { CCCTGTATGCCTCTG- } \\
\text { GTC-3'[18] }\end{array}$ & $\begin{array}{c}\text { 5'-GTCTTTACGGATGT- } \\
\text { CAACG-3'[19] }\end{array}$ \\
\hline
\end{tabular}

Table 1: PCR primers.

\section{Western blot}

The total protein of the tibial bone tissue was extracted, the protein concentration was determined by BCA method, and the $5 \times$ SDS protein was denatured. The 30 ug protein was loaded and electrophoresed. The voltage was first adjusted to $80 \mathrm{v}$ for 30 minutes. After the bromophenol blue dye entered the separation gel, the voltage was adjusted. $120 \mathrm{~V}$, time is about 80 minutes. After electrophoresis, the gel was cut according to the molecular weight of 
the target protein, and the membrane was transferred at a constant current of $200 \mathrm{~mA}$ for $2 \mathrm{~h}$. The PVDF membrane was blocked with $5 \%$ skim milk powder for $1-2 \mathrm{~h}$ at room temperature, and the blocked PVDF membrane was placed in a hybridization solution containing appropriate amount of HIF-1 $\alpha$ antibody (Novus, Littleton, CO) and VEGF antibody (Novus, Littleton, CO). The cells were hybridized overnight at $4{ }^{\circ} \mathrm{C}$ on a shaker, and the membrane was washed 3 times with PBS containing 0.1\% Tween-20 for 10 minutes each time. The membrane was placed in a hybridization solution containing a 1:5000 diluted secondary antibody, and hybridized for 2 hours at room temperature, and the membrane was washed three times with PBS for 10 minutes each time. It was developed in an Image Quant LAS 4000 machine within 15 minutes, and the developer was prepared in a 1:1 ratio for Millipore A and B. The strip image is saved after development, and the strip gray value is analyzed and calculated using the Image Quant LAS 4000 built-in software.

\section{Statistical analysis}

Statistical analysis was performed on the test data using the SPSS18.0 software package. The data were expressed as mean \pm standard deviation and analyzed by one-way analysis of variance (ANOVA). The comparison between the groups was performed by Duncan's test at $\mathrm{P}<0.05$. Significant meaning.

\section{Results}

Mice blood glucose and general conditions

Blood glucose monitoring results (Figure 1) suggest that diabetic mice, diabetes + DFO mice on the first day, 30th day, 60th day of intervention, blood glucose is greater than $300 \mathrm{mg} / \mathrm{dL}$, indicating diabetes The mouse model was successfully established; while the control group had a blood glucose of less than $300 \mathrm{mg} / \mathrm{dL}$. Diabetic mice have more diet, water, and urine, and their body weight is significantly reduced, and hair is messy.

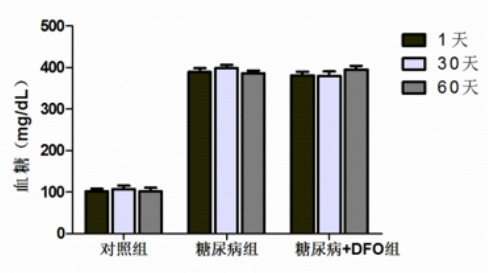

Figure 1: Blood glucose level.

Note: Blood glucose in the diabetic group $(n=10)$ and diabetes+DFO $(n=10)$ group was greater than $300 \mathrm{mg} / \mathrm{dL}$ on the $1 \mathrm{st}, 30$ th, and 60 th day of intervention, indicating that the diabetic mouse model was successfully established.

Annotation: the blood glucose level of the mice in diabete group $(n=10)$ and diabete+DFO $(n=10)$ group was higher than $300 \mathrm{mg} / \mathrm{dL}$, demonstrating a sucessful establishment of diabete mice model.
Micro-CT scan results of cancellous bone of the distal femur of the mouse (Figure 2) and quantitative analysis: as shown in Table 2: BMD, BV/TV, Tb. N, Tb. Th in the diabetic group were significantly lower than the control. Group, while Tb. Sp was significantly higher than the control group. After DFO intervention, BMD, BV/TV, Tb.N, $\mathrm{Tb}$. Th in the diabetic+DFO group were partially improved, and $\mathrm{Tb}$. Sp decreased to some extent. The above indexes were significantly lower than those in the diabetic group $(\mathrm{P}<0.05)$. The difference was significant.

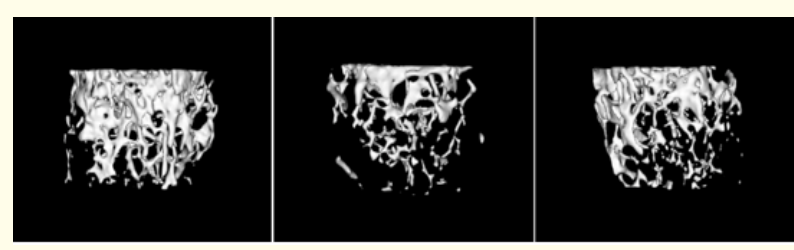

Figure 2: Three-dimensional reconstruction of the canine bone of the femur at the distal end of the mouse.

Note: The cancellous bone of the distal femur of the diabetic group was sparse compared with the control group, while the cancellous bone of the distal femur of the diabetic + DFO group was partially restored compared with the diabetic group.

Annotation: there was less trabecular bone in diabete group compared with control group, and DFO administration resecued the decline in trabecular volume.

\begin{tabular}{|l|ll|l|}
\hline $\begin{array}{l}\text { BMD } \\
\left(\mathrm{mg} / \mathrm{cm}^{3}\right)\end{array}$ & $390.45 \pm 27.98$ & $290.89 \pm 21.01 *$ & $345.39 \pm 27.39 \#$ \\
\hline $\begin{array}{l}\mathrm{BV} / \mathrm{TV} \\
(\%)\end{array}$ & $0.1273 \pm 0.0123$ & $0.0567 \pm 0.0094 *$ & $0.0876 \pm 0.0192 \#$ \\
\hline $\begin{array}{l}\text { Tb.N } \\
(1 / \mathrm{mm})\end{array}$ & $3.7895 \pm 0.2341$ & $2.1227 \pm 0.1733^{*}$ & $2.8964 \pm 0.2156 \#$ \\
\hline $\begin{array}{l}\text { Tb.Th } \\
(\mathrm{mm})\end{array}$ & $0.0402 \pm 0.0012$ & $0.0236 \pm 0.0009 *$ & $0.0342 \pm 0.0014 \#$ \\
\hline $\begin{array}{l}\text { Tb.Sp } \\
(\mathrm{mm})\end{array}$ & $0.2684 \pm 0.0278$ & $0.4782 \pm 0.0321 *$ & $0.3591 \pm 0.0268 \#$ \\
\hline
\end{tabular}

Table 2: Micro-CT quantitative analysis results of canine femur at the distal femur.

${ }^{*} \mathrm{P}<0.05$ in the diabetic group compared with the control group; $\# \mathrm{P}<0.05$ in the diabetic+DFO group compared with the diabetic group.

\section{Three-point bending test}

The results (Figure 3) suggest that the maximum femoral load, maximum bending stress and fracture energy of diabetic mice are lower than those of the control group, which indicates that the femoral mechanical properties of diabetic mice are worse than those of the control group; after DFO intervention The mechanical proper- 
ties of the femur were partially restored, and the maximum load, maximum bending stress and fracture energy were significantly higher than those in the diabetic group $(\mathrm{P}<0.05)$.
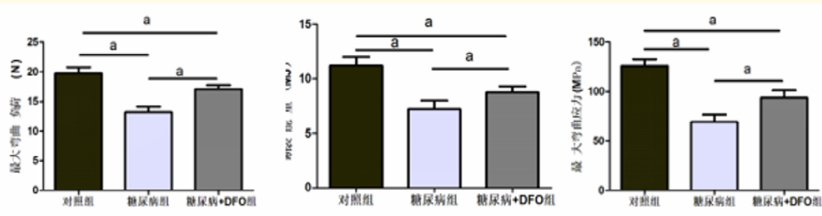

Figure 3: Biomechanical detection of three-point bending of the femur in mice.

Note: The maximum bending stress, maximum bending load and fracture energy of diabetic mice were significantly lower than those of the control group. The maximum bending stress, maximum bending load and fracture energy of diabetic mice were significantly improved after DFO intervention. $\mathrm{P}<0.05$ between groups with $\mathrm{a}, \mathrm{n}=4$ for each group

Annotation: ultimate stress, ultimate load and energy to faliure of femurs in diabete group were significantly lower than control group, and DFO administration significantly increased ultimate stress, ultimate load and energy to faliure of femurs. The group designated with an "a" test significant Differences, $\mathrm{P}<0.05, \mathrm{n}=4$.

\section{Hematoxylin-eosin staining}

The results of hematoxylin-eosin staining (Figure 4) suggest that a large number of osteoblasts adhere to the surface of the cancellous bone of the control group, while the bone of the cancellous bone of diabetic mice is osteogenesis. There were fewer cells, and the number of osteoblasts on the surface of cancellous bone recovered after DFO intervention.

\section{Realtime-PCR}

In the diabetic group, the osteogenic index Runx-2 and osteocalcin mRNA in the diabetic group were significantly lower than those in the control group, and the bone resorption index TRAP mRNA was also lower than the control group; while the diabetes+DFO group Runx-2, Osteocalcin and TRAP were significantly higher than the diabetic group, but lower than the control group. The comparison between the three groups was $\mathrm{P}<0.05$, and the difference was significant.

\section{Westernblot}

The expression of HIF- $1 \alpha$ and VEGF protein in the tibia bone of the diabetic group was lower than that of the control group in the diabetic group. The expression of HIF- $1 \alpha$ and VEGF protein in the bone tissue of the diabetic+DFO intervention group was significantly higher than that of the diabetic group. But lower than the control group.

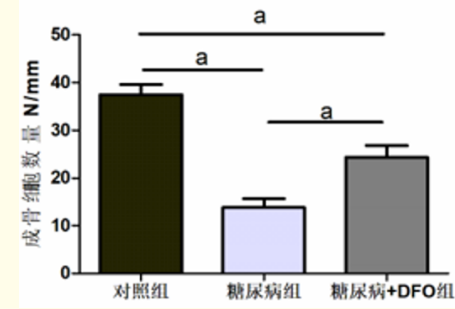

Figure 4: Hematoxylin and eosin staining.

Note: A large number of osteoblasts were attached to the cancellous bone surface of the control group indicated by the black arrow. The osteoblasts on the cancellous bone surface of the diabetic group were less, and the number of osteoblasts was partially restored after DFO intervention. $(\times 400)$.

Note: The number of osteoblasts on the surface of trabecular bone in the control group was significantly higher than that in the diabetic group. The number of osteoblasts on the trabecular surface of the diabetic group increased after application of DFO. $\mathrm{P}<0.05$ was compared between the two groups. Each set of samples was $\mathrm{n}=10$.

Annotation: a large amout of osteoblasts (black arrows pointed) resided along the surface of trabeculae in control group, and the number of osteoblasts decreased significant in diabete group and notcibled by DFO administration. $(\times 400)$. The group designated with an " $a$ " showed significant differences, $\mathrm{P}<0.05, \mathrm{n}=10$.
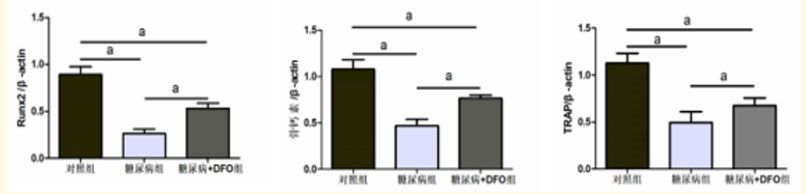

Figure 5: Real-time PCR.

Note: The expression of Runx-2, osteocalcin and TRAP mRNA in the bone tissue of the diabetic group was significantly lower than that of the control group. The expression of Runx-2, osteocalcin and TRAP mRNA in the diabetic+DFO group was significantly higher than that in the diabetic group. $\mathrm{P}<0.05$ between groups. Each set of samples was $n=5$.

Annotation: the mRNA expression levels of Runx-2, osteocalcin and TRAP in diabete group were significanly lower than contral group, and the mRNA expression levels of Runx-2, osteocalcin and TRAP in diabete +DFO group were higher than diabete group, The group designated with an "a" showed significant differences, $\mathrm{P}<0.05, \mathrm{n}=5$. 


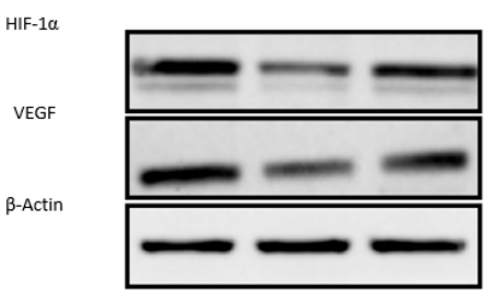

Control group Diabetes group Diabetes + DFO group
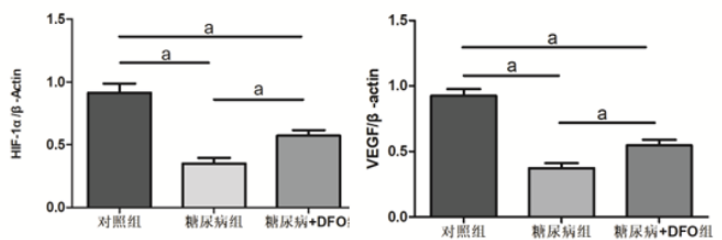

Figure 6: Westernblot.

Note: HIF-1 alpha and VEGF expression in the group of diabetic bone were significantly lower than those in the control group, and HIF-1 alpha and VEGF expression increased significantly after DFO intervention. The band a comparison between the two groups of $\mathrm{P} 0.05$.

Annotation: the expression level of HIF- $1 \alpha$, VEGF in diabete group were lower than control group and was rescued by DFO administration. The group designated with an "a" showed significant differences, $\mathrm{P}<0.05$.

\section{Discussion}

Osteoporosis in type 1 diabetes is mainly related to bone formation disorders. Pathologically, the number of osteoblasts is reduced, the rate of bone formation is decreased, and calcium salt deposition is impeded. At the same time, bone resorption is also reduced due to low bone formation. It leads to a low conversion state of bone metabolism in type 1 diabetes $[2,3,13,14]$. Therefore, promoting bone formation should be a more reasonable method to prevent osteoporosis in type 1 diabetes. Hypoxia-inducible factors play an important role in bone formation: during bone development, hypoxia-inducible factors promote bone growth by up-regulating VEGF, thereby promoting bone growth; hypoxia-inducible factors during bone remodeling Promote accelerated bone remodeling by promoting angiogenesis and promoting bone formation [6,15-19]. Under normal physiological conditions, the bone marrow cavity is in a hypoxic state, and the partial pressure of oxygen is only $1 \%-7 \%$. Therefore, various cells in the bone marrow cavity constantly express HIF- $1 \alpha$; at the same time, hormone-like substances such as insulin-like growth factor-I can also be used frequently. HIF-1 $\alpha$ is stabilized under oxygen conditions. However, in a hyperglycemic environment, even under hypoxic conditions, the expression of HIF- $1 \alpha$ is decreased; in addition, insulin-like growth
factor-I deficiency further degrades the hypoxia-inducible factor signaling pathway $[10,11,16,20]$. Therefore, the activation of hypoxic signaling pathways in theory is conducive to the recovery of normal bone metabolism in patients with type 1 diabetes. Deferoxamine (DFO) is an iron chelator commonly used in clinical hematology. It has been found that DFO chelate iron ions to prevent degradation of HIF- $1 \alpha$ by proline hydroxylase, thereby activating hypoxic signaling pathways under normoxic conditions. Experiments have found that local or systemic application of DFO can promote bone formation and angiogenesis, thereby promoting fracture healing, bone defect repair, and preventing bone loss caused by aging $[7,21$ 23].

Based on the above research background, the experiment attempted to use DFO to intervene in type 1 diabetic mice to observe their effects on bone. The results suggest that: DFO can improve bone mineral density, bone mass and bone structure in diabetic mice to some extent, and the mechanical properties are also partially improved; this indicates that DFO can prevent the formation of osteoporosis in type 1 diabetes to a certain extent; In addition to estrogen-deficient osteoporosis and senile osteoporosis, the third type of osteoporosis that DFO can control in the experiment $[7,23]$. Histological results showed that the number of osteoblasts on the surface of cancellous bone increased significantly after DFO intervention. Runx-2 is a key transcription factor in the process of osteoblast differentiation. Low osteocalcin level is one of the main causes of bone formation in patients with type 1 diabetes. Runx- 2 and osteocalcin mRNA levels were obtained after DFO intervention. Partly increased by $[3,24,25]$. This suggests that the prevention and treatment of osteoporosis in DFO type 1 diabetes mainly contributes to the promotion of "bone formation". The results of our research team have shown that osteoporosis bone metabolism in type 1 diabetes is "low conversion", so the commonly used bone resorption inhibitors in clinical practice may not be suitable for such osteoporosis in principle; currently, bone formation promoting drugs are commonly used. Parathyroid hormone is more expensive; DFO, as a drug already in clinical use, not only promotes bone formation, but also has the advantage of being relatively inexpensive. On the other hand, since DFO can chelate iron ions, DFO also has a certain inhibitory effect on bone resorption [26-28]. However, our results suggest that the bone resorption index, Tartrate-resistant acid phosphatase (TRAP), is increased after DFO intervention. This may be due to increased osteoblasts, increased bone turnover, and enhanced bone resorption. Our previous findings suggest that a reduction in the number of bone vessels in type 1 diabetes is also an important factor leading to a decline in bone formation [2,22]. The results of this experiment indicate that DFO can significantly increase VEGF levels in bone tissue by activating hypoxic signaling pathways, which is beneficial to bone formation recovery. 
There are certain defects in the experiment: 1 . Lack of DFO on the effects of mesenchymal stem cells, osteoblasts, osteoclasts and other cells in high glucose environment and its mechanism. 2. There was no interventional positive control group such as osteoclast inhibitor and bone formation promoter.

\section{Conclusion}

In summary, the experimental results suggest that DFO can prevent the formation of osteoporosis in type 1 diabetic mice to some extent, and its mechanism may be related to the increase of bone formation after activation of hypoxia signaling pathway. Further, the specific molecular mechanism remains to be determined. follow up research.

\section{Bibliography}

1. Armas LA., et al. "Trabecular bone histomorphometry in humans with Type 1 Diabetes Mellitus". Bone 50.1 (2012): 91-96.

2. Peng J., et al. "Low bone turnover and reduced angiogenesis in streptozotocin-induced osteoporotic mice". Connective Tissue Research 57.4 (2016): 277-289.

3. Lu H., et al. "Diabetes interferes with the bone formation by affecting the expression of transcription factors that regulate osteoblast differentiation". Endocrinology 144.1 (2003): 346352.

4. Hie M., et al. "Insulin-dependent diabetes mellitus decreases osteoblastogenesis associated with the inhibition of Wnt signaling through increased expression of Sost and Dkk1 and inhibition of Akt activation". International Journal of Molecular Medicine 28.3 (2011): 455-462.

5. Wang A., et al. "Hyperglycemia diverts dividing osteoblastic precursor cells to an adipogenic pathway and induces synthesis of a hyaluronan matrix that is adhesive for monocytes". Journal of Biological Chemistry 289.16 (2014): 11410-11420.

6. Wang Y., et al. "The hypoxia-inducible factor alpha pathway couples angiogenesis to osteogenesis during skeletal development". Journal of Clinical Investigation 117.6 (2007):16161626.

7. Kusumbe AP., et al. "Coupling of angiogenesis and osteogenesis by a specific vessel subtype in bone". Nature 507.7492 (2014): 323-328.

8. Liu X., et al. "Prolyl hydroxylase inhibitors protect from the bone loss in ovariectomy rats by increasing bone vascularity". Cell Biochemistry and Biophysics 69.1 (2014): 141-149.

9. Peng J., et al. "Dimethyloxalylglycine Prevents Bone Loss in Ovariectomized C57BL/6J Mice through Enhanced Angiogenesis and Osteogenesis". PLoS One 9.11 (2014): e112744.
10. Chen H., et al. "Upregulating Hif-1alpha by Hydrogel Nanofibrous Scaffolds for Rapidly Recruiting Angiogenesis Relative Cells in Diabetic Wound". Advanced Healthcare Materials 5.8 (2016): 907-918.

11. Grewal BS., et al. "Evaluating effects of deferoxamine in a rat tibia critical bone defect model". Journal of Orthopaedic 11.1 (2014): 5-9.

12. Botolin S., et al. "Increased bone adiposity and peroxisomal proliferator-activated receptor-gamma2 expression in type I diabetic mice". Endocrinology 146.8 (2005): 3622-3631.

13. Botolin S and McCabe LR. "Bone loss and increased bone adiposity in spontaneous and pharmacologically induced diabetic mice". Endocrinology 148.1 (2007):198-205.

14. Weng T., et al. "Inactivation of Vhl in osteochondral progenitor cells causes high bone mass phenotype and protects against age-related bone loss in adult mice". Journal of Bone and Mineral Research 29.4 (2014): 820-829.

15. Botusan IR., et al. "Stabilization of HIF-1alpha is critical to improve wound healing in diabetic mice". Proceedings of the National Academy of Sciences of the United States of America 105.49 (2008):19426-19431.

16. Rankin EB., et al. "The HIF signaling pathway in osteoblasts directly modulates erythropoiesis through the production of EPO". Cell 149.1 (2012): 63-74.

17. Stegen S., et al. "Bringing new life to damaged bone: the importance of angiogenesis in bone repair and regeneration". Bone 70 (2015): 19-27.

18. Quinlan E., et al. "Hypoxia-mimicking bioactive glass/collagen glycosaminoglycan composite scaffolds to enhance angiogenesis and bone repair". Biomaterials 52.1 (2015): 358-366.

19. Catrina SB., et al. "Hyperglycemia regulates hypoxia-inducible factor-1alpha protein stability and function”. Diabetes 53.12 (2004): 3226-3232.

20. Jia P., et al. "Deferoxamine released from PLGA promotes healing of osteoporotic bone defect via enhanced angiogenesis and osteogenesis". Journal of Biomedical Materials Research 104.10 (2016).

21. Shen X., et al. "Prolyl hydroxylase inhibitors increase neoangiogenesis and callus formation following femur fracture in mice". Journal of Orthopaedic Research 27.10 (2009):1298-1305.

22. Wang L., et al. "Synergistic protection of bone vasculature and bone mass by desferrioxamine in osteoporotic mice". Molecular Medicine Reports 16.5 (2017): 6642-6649. 
23. Chung JH., et al. "Deferoxamine promotes osteoblastic differentiation in human periodontal ligament cells via the nuclear factor erythroid 2-related factor-mediated antioxidant signaling pathway". Journal of Periodontal Research 49.5 (2014): 563-573.

24. Jia P., et al. "Ferric ion could facilitate osteoclast differentiation and bone resorption through the production of reactive oxygen species". Journal of Orthopaedic Research 30.11 (2012):1843-1852.

25. Kang H., et al. "Desferrioxamine reduces ultrahigh-molecularweight polyethylene-induced osteolysis by restraining inflammatory osteoclastogenesis via heme oxygenase-1". Cell Death and Disease 7.10 (2016): e2435.

26. Edelmayer M., et al. "Effect of prolyl hydroxylase inhibitorloaded collagen barrier membranes on osteoclastogenesis and osteoblastogenesis". Journal of Biomaterials Applications 31.10 (2017): 1370-1379.

27. Drager J., et al. "Local delivery of iron chelators reduces in vivo remodeling of a calcium phosphate bone graft substitute". Acta Biomaterialia 42 (2016): 411-419.

28. Xie W., et al. "Extracellular Iron is a Modulator of the Differentiation of Osteoclast Lineage Cells". Calcified Tissue International 98.3 (2016): 275-283.

Volume 2 Issue 12 December 2019

(C) All rights are reserved by Jia Peng., et al. 